\title{
Collaborative Workforce, Business Process Crowdsourcing as an Alternative of BPO
}

\author{
Gioacchino La Vecchia ${ }^{1}$ and Antonio Cisternino ${ }^{2}$ \\ ${ }^{1}$ CrowdEngineering inc., 440 N.Wolfe Rd., Sunnyvale CA 94085 United States \\ gio@crowdengineering.com \\ ${ }^{2}$ Dipartimento di Informatica, L.go B.Pontecorvo 3, 56127 Pisa \\ cisterni@di.unipi.it
}

\begin{abstract}
Crowdsourcing is the act of outsourcing activities to networked people. This paper presents Business Process Crowdsourcing, an alternative to Business Process Outsourcing where crowd activities are coordinated, work force contributions not wasted and final result guaranteed. The positioning paper shows how to transform canonical business processes in crowdsourced business processes where Web 2.0, social networks, and business process management are combined to deploy business critical process to the Internet, getting the same level of quality and control of traditional outsourcing approaches with conventional workforce.
\end{abstract}

Keywords: crowdsourcing, social production, collaborative intelligence, business process management.

\section{Introduction}

Crowdsourcing, term coined by Howe [2], is the act of taking a job traditionally performed by a designated agent (usually an employee) and outsourcing it to an undefined, generally large group of people in the form of an open call [1].

Crowdsourcing is rapidly becoming a common tool for addressing problems in organizations and businesses, finding always new applications and often exploiting social network to accomplish tasks in a human-wise way rather than resorting to smart algorithms. The technique has been applied with special purpose systems since 2001, for instance to ask hobbyist astronomers to classify Mars craters [3], though it slowly emerged as a more general pattern. Wikipedia [4] has shown how a product built through a collaborative effort by thousands of spontaneous contributors can surpass a linearly organized process performed by professional editors over several years, affecting significantly the encyclopedia business. Crowdsourcing has also been used in several applications, and many game with purpose are played today to exploit the work otherwise wasted in gaming. Fold It!, for instance, is a game with purpose [5] that already contributed to define and implement new synthetic proteins. Among several other areas of application, it is worth citing R\&D managed as open calls over the Internet like Innocentive [6], product design or marketing and communication concept development like Threadless [7], new business concepts development like Quirky [8], product and service testing like uTest [9]. Amazon has been one of the 
first actors in the business market that tried to exploit the workforce in a more systematic way with the Mechanical Turk [10], a system introducing a crowdsourcing task marketplace for applications that need many contributors.

Crowdsourcing problem can be abstracted as follows: a task $T$ can be split into a number of sub-tasks $t_{i}$ that can be assigned to one or more workers $w_{i}$ taken from a set of users $W$. The crowdsourcing system is responsible to generate the associations $\left(w_{i}\right.$, $t_{j}$ ) so that the task $T$ can be accomplished ensuring that sub-tasks are completed, rescheduling in case of failure. A special purpose crowdsourcing system is a system $C_{T}(W)$ designed specifically to associate workers to tasks for a specific task $T$. A general purpose crowdsourcing system $C(T, W)$ is capable of orchestrating and book keeping the whole process being parametric in both $T$ and $W$. A set of constraints $K$, may permit or forbid specific associations among sub-tasks and workers (for instance because a certain micro-task requires a specific knowledge). The various crowdsourcing systems are characterized in the way $C, T, K$, and $W$ are chosen; for instance, if workers are not trusted the system may associate more workers with the same subtask, adopting a majority vote among the results produced.

Crowdsourcing is the natural evolution of outsourcing in the era of social networks giving better economical returns also where outsourcing is already in place, enabling also small/medium enterprises and small institutions to get benefits from crowdsourcing internal processes. Several existing vertical crowdsourcing sites have not been able to fully address the market of outsourced or to-be-outsourced business processes. The main reason for this partial result is to be found in the reluctance of large enterprises in outsourcing activities, without maintaining the control of the process because this could seriously compromise their core business.

\section{Today's Crowdsourcing Scenario}

Crowdsourcing is today well known as a cheap alternative to hire professionals; it may appear in the form of a contest to which several apply to propose their solution or their contribution, in answer to a call over the Internet. In marketing applications creative professionals propose a logo or an idea for an advertising spot and a group of experts, usually company internals, decide who the winner is.

This type of crowdsourcing, which we define "contest", is clearly a waste of workforce: for a single price winner the work of many others is overlooked. It could work only assuming that large crowds of contributors compete for a limited numbers of contests. Besides, if the number of tasks to outsource is large, talented people will unlikely waste their time with a low chance to get some reward.

Another kind of crowdsourcing that is becoming popular is the "task marketplace", like Amazon Mechanical Turk, where several simple tasks are posted by companies or individuals These tasks are usually repetitive actions like tagging photos or moderating contents, or simple parts of a larger problem like translating a section of a document or answer questions of a questionnaire. Because of the simple task nature, the reward in these cases is low since it doesn't require special abilities, thus the set $W$ is large implying a large offer of workers.

A family of crowdsourcing systems that is successfully working is the one in which the set $W$ is constituted by experts are associated with complex problems submitted by users and the system associate tasks attempting to find the best professional 
to fulfill the task at the lowest cost. For professionals this is a great resource to find new assignments and be paid organizing autonomously their work; we will refer to this model as "bid".

\section{Crowdsourcing and BPO}

Is the crowdsourcing, as we know it today, a real alternative to outsourcing for large enterprises? Let's take in consideration the crowdsourcing scenarios we described in the previous section from the point of view of a large enterprise or institutions willing to crowdsource their internal business processes.

The contest crowdsourcing model is something that applies to specific niches of business processes. Let us consider as example a marketing department: a contest can be started on a company's own site or in a crowdsourcing site to select a new logo or a new design for a package; while the creative work is performed by crowdsourcing contributors a lot of work is left to the company; in the first place the selection process and the winner evaluation. Often this type of crowdsourcing is used to generate buzz and to stimulate discussions about a company or a brand and so enable cheap user generated marketing. However, this approach is not cost effective to handle complex internal business processes.

Task marketplace crowdsourcing is a great resource for processes compatible with a divide et impera solution approach. If you have a document to translate you can split it in several sections and post each section as a task to complete (to translate) and wait for the contributions. This works well in phase of preparation for simple processes where the split in single tasks is relatively simple. But still a significant effort is needed in order to monitor the contributors' work, the completion of the tasks, merging of the single parts, and the control of the quality of the final deliverable. It could help in some cases but again if the complexity of the business process or the need of a fine control and quick reaction to some event apply then it would be a mess to manage it in such a way.

Bid crowdsourcing is something not new to large companies; the main difference is that now there exist communities of experts, while some time ago companies already collaborating with the enterprise looking for help were invited to submit bids. In this kind of crowdsourcing the effort is both in the preparation, execution, selection, and acceptance phases. In a software project, for instance, it must be paid attention when writing the requirements, in order to avoid misunderstanding in the economic evaluation; moreover each submitted proposal should be carefully read.

In summary we think that currently available crowdsourcing models are able to address only a small percentage of the business process of an enterprise where the involvement of the crowd could bring benefits. The reasons are that core business processes are usually quite complex, need to be integrated with other company's business processes and eventually with software platforms supporting them. So orchestration of resources, control of the processes in term of time, delivery and quality are key requirements to enlarge the usage of crowdsourcing in the enterprise. So a new model is necessary to fully exploit the power of crowdsourcing and we'll call it Business Process Crowdsourcing. 


\section{Crowdsourcing in Customer Service}

In a traditional customer service model requests are managed by internal Customer Assistants or by Outsourcing operators. When a new request $R$ is asked it is assigned or taken in charge by a worker $w_{i}$. Assignment to a specific worker is performed in the most advanced call centers based on the type of request, expertise of the worker and value of the customer. Some of the typical challenges for a contact center are to maintain a level of expertise among assistants good enough to properly answer request at the first contact and to adequately size the workforce to face the intraday volume of requests incoming.

Crowdsourcing can help a lot here, the expertise distributed among users, in particular among customers of the same company providing support, is large enough to provide a good level of support. A widely used rule says that when you have an online community you can expect $90 \%$ of users to be passive, $9 \%$ to be intermittent contributors and $1 \%$ to be heavy contributors [11]. Online members are already a fraction of total customer base; you can expect to have between a $10 \%$ and $50 \%$ of total customer base to use online channels depending on the industry and the affinity of the service or product supported with an online presence. So if we take in consideration a 10 million users Telco customr base and we apply a conservative rate of $20 \%$ of users going online we have a 2 million community where 200k users contribute and 20k among them are heavy contributors.

A successful application of crowdsourcing here can change the economics of the call center. A $20 \%$ of deflection of requests from the contact center to the community is an extraordinary result for cost reduction. And by transforming voice calls requests in recorded messages for the community deflection rate can raise even more. At this point the challenge is not anymore in migrating users from voice to online channels but to make sure that community experts will be able to manage all those requests.

To achieve this result a fine grain crowdsourcing process must be in place deeply integrated with company enterprise platforms. The model applied must be very similar to the way complex enterprise processes are managed but considering the key differences of this workforce, because they are usually voluntary and must be rewarded, they are not under a contract but their performance must be evaluated, they are not respecting scheduled shifts of work nut must be orchestrated and more their skill and ability to support must be evaluated day by day in order to involve the best(s) expert for each request. We'll define Business Process Crowdsourcing that crowdsourcing model taking in consideration all of these elements of the problem of outsourcing a complex internal business process to the crowd.

\section{Cloud of Crowd}

A natural convergence of crowdsourcing techniques is toward cloud computing. Cloud computing is a business model in which large computing infrastructures are made available to third party that have need for computing resources. Crowdsourcing shares many similarities with this business model and may be affected by the attention it will receive in the future. It is possible to define a cloud specifically designed for general purpose crowdsourcing in which the set of workers $W$ is shared among different tasks $T_{k}$. In this way businesses may deploy new crowdsourcing services in an 
elastic way, so that specific crowdsourcing services may be used without having to deploy a specific infrastructure.

If cloud services depend only from computational resources and software available on nodes, a cloud of crowd also depends on the know-how of the users working at the various sub-tasks, introducing constraints on the type of tasks that can be served by a specific cloud. Moreover, since the knowledge may vary among workers, it is responsibility of the system to find the optimal match between workers and sub-tasks and keep track of who did what.

Traditional outsourcing is a coarse grain model for externalizing a service reducing internal costs; however it imposes overhead because services are dimensioned to handle potential spikes. Cloud of crowd allows for a finer grain outsourcing mechanism, in which it is possible to track every single sub-task delivered introducing a pay-per use model. This is the dual problem with respect to micro-payments: work should be tracked with a very fine grained model, imposing that the tracking cost does not surpass the value generated by the worker. It should be noted, however, that human workers may perform tasks that computers may not be able to solve (for instance finding nicer objects of a given example) or solve it easily; therefore the value generated by a single worker may relevant with respect to the system. Moreover, if the set $W$ is large enough the probability that a sub-task is quickly assigned to a worker is high.

\section{Summary}

A new model of crowdsourcing is needed in order to graduate crowd involvement for enterprise business processes. Enterprise crowdsourcing is so possible but needs to be supported in the same way an enterprise business process is with all the distinctions considering the different nature of the workforce involved.

Deeply different from existing crowdsourcing models enterprise crowdsourcing must ensure that no resources are wasted, best contributor available is assigned to each single task, performances are carefully evaluated to build dynamic skill profiles and to reward contributors, a strong business process control ensure that unattended tasks are completed respecting the service levels in place, quality visibility points are constantly managed and monitored, involvement of the crowd and communication with them are facilitated in order to make the job easier and when possible amusing.

\section{References}

1. Crowdsourcing Blog, http://www.crowdsourcing.com/ (Last accessed 20/6/2010 )

2. Howe, J.: The rise of Crowdsourcing. WIRED Magazine (June 14, 2006)

3. Britt, R.R.: Nasa Wants you...to identify Martians craters (February 2001), http: / / www. space.com/scienceastronomy/solarsystem/ click_workers_010202.html (Last accessed: 20/6/2010)

4. Wikipedia: Crowdsourcing, http: / / en.wikipedia.org/wiki / Crowdsourcing (Last accessed: 20/6/2010)

5. Fold.it!, http: // fold.it/ (Last accessed: 20/6/2010) 
6. Innocentive, http: / / www . innocentive. com/ (Last accessed: 20/6/2010)

7. Threadless, http: //www. threadless . com/ (Last accessed: 20/6/2010)

8. Quirky, http: / /www . quirky . com/ (Last accessed: 20/6/2010)

9. UTest, http: / /www . utest. com/ (Last accessed: 20/6/2010)

10. Amazon Mechanical Turk, https : / / www . mturk. com/ (Last accessed: 20/6/2010)

11. Nielsen, J.: Participation Inequality: Encouraging More Users to Contribute, http://www.useit.com/alertbox/participation_inequality.html (Last accessed: 20/6/2010) 